\title{
Oriented Walk Double Covering and Bidirectional Double Tracing
}

\author{
Fan Hongbing \\ Mathematics Department of Shandong University \\ Jinan Shandong, 250100, P.R.China \\ Email: hfan@sdu.edu.cn; Fax: 86-531-8902167 \\ Xuding Zhu* \\ Department of Applied Mathematics \\ National Sun Yat-sen University \\ Kaohsiung, Taiwan 80424 \\ Email: zhu@math.nsysu.edu.tw; Fax: 886-7-5253809
}

\begin{abstract}
An oriented walk double covering of a graph $G$ is a set of oriented closed walks which, traversed successively, combined will have traced each edge of $G$ once in each direction. A bidirectional double tracing of a graph $G$ is an oriented walk double covering which consists of a single closed walk. A retracting in a closed walk is the immediate succession of an edge by its inverse. Every graph with minimum degree 2 has a retracting free oriented walk double covering and every connected graph has a bidirectional double tracing. The minimum number of closed walks in a retracting free oriented
\end{abstract}

*This research was supported in part by the National Science Council of the Republic of China under grant NSC87-2115-110-004. 
walk double covering of $G$ is denoted by $c(G)$. The minimum number of retractings in a bidirectional double tracing of $G$ is denoted by $r(G)$. We shall prove that for all connected non-cycle graphs $G$ with minimum degree at least $2, r(G)=c(G)-1$. The problem of characterizing those graphs $G$ for which $r(G)=0$ was raised by Ore. Thomassen solved this problem by relating it to the existence of certain spanning trees. We generalize this result, and relate the parameters $r(G), c(G)$ to spanning trees of $G$. This relation yields a polynomial time algorithm to determine the parameters $c(G)$ and $r(G)$.

\section{Introduction}

An oriented walk double covering of a graph $G$ is a set $\mathcal{C}$ of oriented closed walks whose union traverses each edge of $G$ exactly once in each direction. If $\mathcal{C}$ consists of a single closed walk $W$, it is called a bidirectional double tracing. A retracting in a walk $W$ is the immediate succession of an edge by its inverse, as in the form $W=$...eve..., where edge $e$ is called a retracting edge and $v$ a retracting vertex. We denote by $r(W)$ the number of retractings of $W$. It is easy to see that every connected graph $G$ has a bidirectional double tracing [3]. Indeed, replacing each edge of $G$ by two opposite arcs would result in an Eulerian digraph. The Euler tour of this digraph is a bidirectional double tracing of $G$. We denote by $r(G)$ the minimum number of retractings in a bidirectional double tracing of $G$.

In 1951, Ore [6] raised the problem of characterizing those graphs which admit a retracting-free bidirectional double tracing, i.e., those graphs $G$ for which $r(G)=0$. This problem was studied by Fleischner [3], Eggleton and Skilton [2], etc. The following theorem of Thomassen [8] gives a solution:

Theorem 1 A connected multigraph $G$ has a retracting-free bidirectional double tracing if and only if $G$ has no vertex of degree 1 and has a spanning tree $T$ such that each connected component of $G-E(T)$ has an even number of edges or contains a vertex $v$ which has degree $d_{G}(v) \geq 4$.

In this paper, we investigate the parameter $r(G)$ for connected graphs $G$, as 
well as constructions of bidirectional double tracings $W$ for which $r(W)=r(G)$. In Lemma 1, we observe that any graph $G$ with minimum degree $\delta(G) \geq 2$ has a retracting free oriented walk double covering. We denote by $c(G)$ the minimum number of closed walks in a retracting free oriented walk double covering of $G$. It follows from the definition that $r(G)=0$ if and only if $c(G)=1$. We shall prove that $r(G)=c(G)-1$ for all connected non-cycle graphs with minimum degree $\delta(G) \geq 2$. Then we generalize Thomassen's result and relate the two parameters $r(G)$ and $c(G)$ to the structures of spanning trees of $G$. Suppose $T$ is a spanning tree of a connected graph $G$. We denote by $o(T)$ the number of components of $G-E(T)$ with odd number of edges and with no vertex of degree (in $G$ ) greater than 3 , we refer such a component as an odd component of $G-E(T)$; and denote by $o(G)$ the minimum of $o(T)$ among all spanning trees $T$ of $G$. Theorem 1 is equivalent to say that, for a connected graph $G$ with $\delta(G) \geq 2, r(G)=0$ if and only if $o(G)=0$. We generalize this result and prove that $r(G)=o(G)$ for all connected graphs with minimum degree $\delta(G) \geq 2$. The original proof of Thomassen's result transform the existence of retracting free bidirectional double tracing of a graph $G$ to the strictly upper embeddability of $G$. Our proof use induction on the number of edges. The case $r(G)=0$ provides an alternate proof of Thomassen's result. Using our proof, the retracting free bidirectional double tracing can be constructed directly from the spanning tree $T$ for which $o(T)=0$.

Thomassen [8] also gave a polynomial time algorithm to determine whether or not there exists a spanning tree with the above property, by reducing the problem to the existence of perfect matching in 2-polymatroids. We shall use the same idea to give an algorithm to determine the parameters $c(G)$ and $r(G)$ in time polynomial in size of $G$. There is a minor error in Thomassen's proof, which we shall explain and which is corrected in the modified argument presented in this paper.

We shall use terminology and notation of Bondy and Murty [1]. All graphs considered here are finite, but may have loops and multiple edges. A loop on a vertex $v$ contributes 2 to the degree of $v$. A spanning tree of $G$, of course, contains no loops. For two walks $X, Y$ of a graph $G$ such that the last vertex of $X$ is equal to the first vertex of $Y$, we shall denote by $X Y$ the concatenation of $X$ and $Y$, which is again a walk of $G$. When we consider the parameter $r(G)$, we may assume 
that the graph $G$ has minimum degree at least 2 , because we can add a loop to each vertex of degree 1 , and this does not change the retracting number. Another operation frequently used is the contraction of edges. Suppose $e=a b$ is an edge of $G$. We shall denote by $G$.e the graph obtained from $G$ by contracting the edge $e$, i.e., by deleting the edge $e$, and identifying its two end vertices $a$ and $b$. Obviously, if an edge $e$ is incident to a vertex of degree 2, then $r(G . e)=r(G)$. By successively contracting the edges that incident with a vertex of degree 2 , we obtain a graph with minimum degree at least 3 , denoted by $G^{*}$. It is obvious that $G^{*}$ is uniquely determined by $G$, up to an isomorphism. So given a connected graph $G$ not a cycle we can by addition of a loop to each degree 1-vertex and successive contractions, one by one, of each edge incident with a degree 2-vertex construct a graph $H$ with $\delta(H) \geq 3$ and $r(H)=r(G)$.

\section{Some preliminary results}

A rotation scheme of a graph $G$ with vertices $\left\{v_{1}, v_{2}, \cdots, v_{n}\right\}$ is a collection $\left\{\pi_{1}, \ldots, \pi_{n}\right\}$ such that each $\pi_{i}$ is a permutation on the edges incident with $v_{i}$, for $i=1, \ldots, n$. Each rotation scheme corresponds to an oriented walk double covering, which consists of the orbits $e_{1} v_{j} e_{2} v_{k} e_{3} \ldots$, where $\pi_{j}\left(e_{1}\right)=e_{2}=v_{j} v_{k}, \pi_{k}\left(e_{2}\right)=e_{3}$, etc. Conversely, each oriented walk double covering corresponds to a rotation scheme.

Lemma 1 Every graph $G$ of minimum degree at least two has a retracting free oriented walk double covering.

Proof. For every vertex $v$ of $G$, choose a permutation $\pi_{v}$ which does not fix any edge. Then the orbits of the rotation scheme forms a retracting free oriented walk double covering of $G$.

Lemma 2 Suppose $G$ is a graph and $\mathcal{C}$ is a retracting free oriented walk double covering of $G$. If $v$ is a vertex of $G$ of degree at least 4 , then all the walks in $\mathcal{C}$ passing through $v$ can be combined into a single retracting free walk. 
Proof. For each closed walk $W \in \mathcal{C}$ which passes through $v$, we cut $W$ at vertex $v$ and obtain pieces of $W$. Each of the pieces is a walk which starts at $v$ and ends at $v$, but having all other vertices are distinct from $v$. (These walks are actually closed walks, each passing through $v$ exactly once and may contain retractings at $v$. However we would rather consider them as walks with initial and terminal vertices equal to $v$, as we shall paste them together into a single closed walk later.) Suppose $v$ has degree $k \geq 4$. By applying this cutting operation to all the walks of $\mathcal{C}$ that pass through $v$, we obtain $k$ walks, all of which have initial vertices and terminal vertices equal $v$. Let $W_{1}, W_{2}, \cdots, W_{k}$ be the $k$ walks. Let $D$ be the directed graph with vertices $W_{1}, W_{2}, \cdots, W_{k}$ and in which $\left(W_{i}, W_{j}\right)$ is an arc if and only if $i \neq j$ and the terminal edge of $W_{i}$ is not the reverse of the initial edge of $W_{j}$. Thus each vertex of $D$ has out-degree at least $k-2$ and in-degree at least $k-2$. It is easy to see that $D$ has a directed Hamiltonian cycle $H$ if $k \geq 4$. Paste the walks $W_{1}, W_{2}, \cdots, W_{k}$ together in such a way that the initial edge of $W_{j}$ succeeds the terminal edge of $W_{i}$ when $\left(W_{i}, W_{j}\right)$ is an arc of the Hamiltonian cycle $H$. It follows from the definition of $D$ that the resulting closed walk is retracting free, and combines all the walks passing through $v$ into a single closed walk.

Corollary 1 Suppose $G$ is a connected graph with $\delta(G) \geq 2$. If $G$ has at most one vertex of degree 2 or 3 , and the vertex of degree 2 or 3 has no loop on it, then $r(G)=0$.

Proof. By Lemma 1, $G$ contains a retracting free oriented walk double covering. Applying Lemma 2 to all vertices of $G$ of degree at least 4, we obtain a retracting free oriented walk double covering, say $\mathcal{C}$, such that for every vertex $u$ of degree at least 4 , there is only one closed walk of $\mathcal{C}$ passing through it. If $\mathcal{C}$ contains two or more closed walks, then all these closed walks only intersect at the vertex, say $v$, which has degree 2 or 3 . This is possible only if $v$ has a loop on it. Therefore $\mathcal{C}$ consists of a single closed walk, and hence it is a bidirectional double tracing of $G$.

Lemma 3 Let $G$ be a connected graph with $\delta(G) \geq 2$, then the following statements hold: 
1. If $G^{\prime}$ is a subdivision of $G$, then $r(G)=r\left(G^{\prime}\right), c(G)=c\left(G^{\prime}\right), o(G)=o\left(G^{\prime}\right)$.

2. If e is a nonloop edge of $G$, then $r(G . e) \leq r(G), c(G . e) \leq c(G), o(G . e) \leq o(G)$.

3. If $e$ is not a cut edge of $G$ and both end vertices of e has degree at least 3, then $r(G-e)-1 \leq r(G) \leq r(G-e)+1, c(G-e)-1 \leq c(G) \leq$ $c(G-e)+1, o(G-e)-1 \leq o(G) \leq o(G-e)+1$.

4. Let $u, v$ be two vertices of $G$, and $G^{\prime}$ be the graph obtained by identifying $u$ and $v$, then $r\left(G^{\prime}\right) \leq r(G), c\left(G^{\prime}\right) \leq c(G), o\left(G^{\prime}\right) \leq o(G)$.

Proof. We only prove (1), statements (2)-(4) can be proved similarly. Suppose $G^{\prime}$ is obtained from $G$ by replacing the edge $e=u v$ with a path $P=(u, w, v)$. Let $e_{1}=u w$ and $e_{2}=w v$.

Let $W$ be a bidirectional double tracing of $G$ with $r(W)=r(G)$. Then replace in $W$ the segment $u e v$ by $u e_{1} w e_{2} v$, and the segment veu by $v e_{2} w e_{1} u$, we obtain a bidirectional double tracing $W^{\prime}$ of $G^{\prime}$ with $r\left(W^{\prime}\right)=r(G)$. Therefore $r\left(G^{\prime}\right) \leq r(G)$. Conversely, let $W^{\prime}$ be a bidirectional double tracing of $G^{\prime}$ with $r\left(W^{\prime}\right)=r\left(G^{\prime}\right)$. Without loss of generality, we may assume that $W^{\prime}$ has no retraction at $w$. Indeed, if $W^{\prime}$ has a retraction at $w$, then it is of the form $W^{\prime}=X v e_{2} w e_{2} v Y u e_{1} w e_{1} u Z$. (Hence it has two retractions at $w$.) In this case, we may replace $W^{\prime}$ by $W^{\prime \prime}=$ $X Y u e_{1} w e_{2} v e_{2} w e_{1} u Z$. It is obvious that $r\left(W^{\prime \prime}\right) \leq r\left(W^{\prime}\right)$, and hence $r\left(W^{\prime \prime}\right)=r\left(W^{\prime}\right)$, because $r\left(W^{\prime}\right)=r\left(G^{\prime}\right)$. Since $W^{\prime}$ has no retraction at $w, W^{\prime}$ is of the form $W^{\prime}=$ $X u e_{1} w e_{2} v Y v e_{2} w e_{1} u Z$. Then $W=X u e v Y v e u Z$ is a bidirectional double tracing of $G$ with $r(W)=r\left(W^{\prime}\right)$. Therefore $r(G) \leq r\left(G^{\prime}\right)$, and hence $r(G)=r\left(G^{\prime}\right)$.

Let $\mathcal{C}$ be a retracting free oriented walk double covering of $G$. Replace (in any element $C$ of $\mathcal{C}$ ) the segment $u e v$ with $u e_{1} w e_{2} v$, and replace veu with $v e_{2} w e_{1} u$. Then we obtain a retracting free oriented walk double covering of $G^{\prime}$ which contains the same number of closed walks as $\mathcal{C}$. Therefore $c\left(G^{\prime}\right) \leq c(G)$. Conversely, it is also straightforward to show that $c(G) \leq c\left(G^{\prime}\right)$. Therefore $c(G)=c\left(G^{\prime}\right)$. 
Let $T^{\prime}$ be a spanning tree of $G^{\prime}$ with $o\left(T^{\prime}\right)=o\left(G^{\prime}\right)$. Then $T^{\prime}$ contains at least one of the two edges $e_{1}, e_{2}$. Without loss of generality, assume $T^{\prime}$ contains the edge $e_{1}$. Let $T=T^{\prime} \cdot e_{1}$. Then $T$ is (isomorphic to) a spanning tree of $G$. Obviously $o(T) \leq o\left(T^{\prime}\right)=o\left(G^{\prime}\right)$. Hence $o(G) \leq o\left(G^{\prime}\right)$. Conversely let $T$ be a spanning tree of $G$ with $o(T)=o(G)$. If $T$ contains the edge $e$, then let $T^{\prime}$ be obtained from $T$ by subdividing $e$ into a path of length 2 . Then $T^{\prime}$ is (isomorphic to) a spanning tree of $G^{\prime}$ with $o\left(T^{\prime}\right)=o(T)$. Therefore $o\left(G^{\prime}\right) \leq o(G)$, and hence $o\left(G^{\prime}\right)=o(G)$. If $T$ does not contain the edge $e$, then let $T^{\prime}=T+e_{1}$ and let $T^{\prime \prime}=T+e_{2}$. Then it is straightforward to verify that both $T^{\prime}$ and $T^{\prime \prime}$ are spanning trees of $G^{\prime}$, and $\min \left\{o\left(T^{\prime}\right), o\left(T^{\prime \prime}\right)\right\}=o(T)$. Therefore $o\left(G^{\prime}\right) \leq o(G)$ and hence $o\left(G^{\prime}\right)=o(G)$.

Lemma 4 Let $W$ be a closed walk such that no two retracting edges retracting at the same vertex and each retracting vertex is passed by $W$ at least twice. Then $W$ can be decomposed into a set of at most $r(W)+1$ retracting free closed walks.

Proof. We prove this by induction on $r(W)$. It is clearly true when $r(W)=0$. Assume that $r(W)=k>0$ and that the lemma is true for all closed walks $W^{\prime}$ with $r\left(W^{\prime}\right)<k$. Let $e=u v$ be an edge such that $W$ has a retracting at $v$ in the form $W=$ ueveuP $P_{1} v P_{2} u$. Let $W_{1}=v e u P_{1} v, W_{2}=u e v P_{2} u$, then $W_{1}, W_{2}$ are two closed walks and they traverse the the same edges in the same directions as $W$ does, and $r\left(W_{1}\right)+r\left(W_{2}\right)=r(W)-1=k-1$. If, for $i=1,2$, each retracting vertex of $W_{i}$ is passed by $W_{i}$ at least twice, then by the induction hypothesis, $W_{1}, W_{2}$ can be decomposed into a set of at most $r\left(W_{1}\right)+1+r\left(W_{2}\right)+1=r(W)+1$ retracting free closed walks. If, say, $W_{1}$ has a retracting vertex $x$, with retracting edge $f=x y$, which is passed by $W_{1}$ only once, then since $x$ is a retracting vertex of $W$, and by the assumption $x$ is passed by $W$ at least twice, it follows that $W_{2}$ passes through $x$. Let $W_{1}=y f x f y P_{1}^{\prime} y$, and let $W_{2}=x P_{2}^{\prime} x$. Then $W_{3}=y f x P_{2}^{\prime} x f y P_{1}^{\prime} y$ is a closed walk with $r\left(W_{3}\right)=r(W)-2$, and which traverses the the same edges in the same directions as $W$ does. Hence $W_{3}$ passes each retracting vertex at least twice. By the induction hypothesis, $W_{3}$ can be decomposed into a set of at most $r\left(W_{3}\right)+1$ retracting free closed walks. 
Theorem 2 For any connected non-cycle graph $G$ with minimum degree at least 2, we have $r(G)=c(G)-1$.

Proof. Let $W$ be a bidirectional double tracing with minimum number of retractings $r(W)=r(G)$. We may assume that $G$ has minimum degree at least 3 . Then it is easy to see that no two retracting edges of $W$ are retracted at the same vertex. By Lemma $4, W$ can be decomposed into at most $r(W)+1=r(G)+1$ retracting free closed walks. Therefore $c(G) \leq r(G)+1$. Conversely, let $\mathcal{C}$ be a retracting free oriented double walk covering of $G$ with minimum number of closed walks $|\mathcal{C}|=c(G)$. Let $W_{1}, W_{2}$ in $\mathcal{C}$ pass through the same vertex say $v$. By Lemma $2, d_{G}(v)=3$. Thus $W_{1}, W_{2}$ have a common edge $e$ incident with $v$. Then $v W_{1} v W_{2} v$ would be a closed walk with retracting edge $e$.

Since $G$ is connected, we can combine all closed walks in $\mathcal{C}$ into a single closed walk. Each time the number of closed walks decreases by 1 , the number of retractings increases by 1 . Thus the resulting closed walk has $c(G)-1$ retracting edges. Therefore $r(G) \leq c(G)-1$ and hence $r(G)=c(G)-1$.

Theorem 3 Let $G$ be a graph whose vertices are of degrees 2 and 3 , and let $\mathcal{C}$ be a retracting free oriented walk double covering of $G$. Then $|\mathcal{C}| \equiv|E(G)|-|V(G)|$ $(\bmod 2)$.

Proof. If $G$ is a cycle, then it is obvious that the theorem is true. Assume that $G$ contains at least one vertex of degree 3 . Since contracting an edge $e$ does not change the parity of $|E(G)|-|V(G)|$, by applying Lemma 3, we may assume that $G$ has minimum degree at least 3 , and hence $G$ is a cubic graph. We proceed by induction on $|E(G)|$. Assume that $|E(G)|=k$, and that the theorem is true for all graphs $G^{\prime}$ with $\left|E\left(G^{\prime}\right)\right|<k$. Let $e=u v$ be an edge of $G$. Assume that $W_{1} \in \mathcal{C}$ contains uev, and let $W_{2} \in \mathcal{C}$ contain veu.

If $W_{1}=W_{2}=u e v P_{1} v e u P_{2} u$, then since both vertices $u, v$ have degree 3 , the closed walks $W^{\prime}=v P_{1} v$ and $W^{\prime \prime}=u P_{2} u$ are both retracting free. Let $G^{\prime}=G-e$. Then $\mathcal{C}^{\prime}=\left(\mathcal{C} \backslash\left\{W_{1}\right\}\right) \cup\left\{W^{\prime}, W^{\prime \prime}\right\}$ is a retracting free oriented walk double covering of $G^{\prime}$. By the induction hypothesis, we have $\left|\mathcal{C}^{\prime}\right| \equiv\left|E\left(G^{\prime}\right)\right|-\left|V\left(G^{\prime}\right)\right|(\bmod 2)$. 
Obviously $|E(G)|-|V(G)|=\left|E\left(G^{\prime}\right)\right|-\left|V\left(G^{\prime}\right)\right|-1 \quad(\bmod 2)$. Therefore $|\mathcal{C}|=$ $\left|\mathcal{C}^{\prime}\right|-1 \equiv|E(G)|-|V(G)| \quad(\bmod 2)$.

Assume next that $W_{1} \neq W_{2}$. Let $W_{1}=u e v P_{1} u$ and $W_{2}=v e u P_{2} v$. Then $W=v P_{1} u P_{2} v$ is a retracting free closed walk of $G^{\prime}=G-e$, and $\mathcal{C}^{\prime}=(\mathcal{C} \backslash$ $\left.\left\{W_{1}, W_{2}\right\}\right) \cup\{W\}$ is a retracting free oriented walk double covering of $G^{\prime}$. Again, by the induction hypothesis, $\left|\mathcal{C}^{\prime}\right| \equiv\left|E\left(G^{\prime}\right)\right|-\left|V\left(G^{\prime}\right)\right| \quad(\bmod 2)$, which implies that $|\mathcal{C}|=\left|\mathcal{C}^{\prime}\right|+1 \equiv|E(G)|-|V(G)| \quad(\bmod 2)$.

The following result proved in [8] is an easy consequence of Theorem 2 and Theorem 3.

Corollary 2 Suppose $G$ is a connected graph whose vertices are of degrees 2 and 3. If $|E(G)|-|V(G)| \equiv 0(\bmod 2)$, then $r(G)>0$.

Lemma 5 Let $v$ be a vertex of $G$ of degree at least 3. If $r(G-v)=0$, then $r(G)=0$.

Proof. Let $v_{1} P_{1} v_{2} P_{2} v_{3} \ldots v_{k} P_{k} v_{1}$ be a retracting-free bidirectional double tracing of $G-v$, where $v_{1}, \ldots, v_{k}$ are the neighbours of $v$. Let $e_{i}=v v_{i}, i=1, \ldots, k$. Then

$v_{1} e_{1} v e_{k-1} v_{k-1} P_{k-1} v_{k} e_{k} v e_{k-2} v_{k-2} P_{k-2} v_{k-1} e_{k-1} v e_{k-2} \ldots v_{3} e_{3} v e_{1} P_{1} v_{2} e_{2} v e_{k} v_{k} P_{k} v_{1}$

is a retracting-free bidirectional double tracing of $G$.

\section{$3 \quad$ Bidirectional double tracing and spanning trees}

We shall show in this section that $r(G)=o(G)$ for all connected graphs $G$ with $\delta(G) \geq 2$. This generalizes Thomassen's result, which is the case $r(G)=0$. We first give an alternative proof of Thomassen's result, that a connected graph $G$ with $\delta(G) \geq 2$ has $r(G)=0$ if and only if $o(G)=0$.

An alternative proof of Theorem 1. Suppose $r(G)=0$. We shall prove that $o(G)=0$ by induction on $|E(G)|$. By Lemma 3, we may assume that $\delta(G) \geq 3$. 
If $|E(G)|=2$, then $G$ is a vertex with two loops. In this case $r(G)=0$, and $E(T)=\varnothing$ for any spanning tree $T$ of $G$ so $o(G)=0$.

Assume that $|E(G)| \geq 3$ and the above statement is true for graphs $G^{\prime}$ with $\left|E\left(G^{\prime}\right)\right|<|E(G)|$. Let $W$ be a retracting free bidirectional double tracing of $G$.

Suppose there are two edges $e_{1}, e_{2}$ of $G$ incident to a vertex $v$ such that $W$ is of the form $e_{1} v e_{2} P_{1} e_{2} v e_{1} P_{2}$. Then let $G^{\prime}$ be the graph obtained from $G$ by splitting the vertex $v$ into two vertices $v^{\prime}$ and $v^{\prime \prime}$ so that $v^{\prime}$ is incident only to edges $e_{1}, e_{2}$, and that $v^{\prime \prime}$ is incident to all other edges that were incident to $v$. Now $W$ can be viewed as a bidirectional double tracing of $G^{\prime}$, which implies that $r\left(G^{\prime}\right) \leq r(W)=$ $r(G)=0$. Define $G^{*}$ as the graph obtained from $G^{\prime}$ by successively contracting edges which are incident to a vertex of degree 2. Then $G^{\prime}$ is a subdivision of $G^{\prime *}$. Since $G^{\prime}$ does have a vertex of degree 2 , it follows that $E\left(G^{*}\right)<E\left(G^{\prime}\right)=E(G)$. As $r\left(G^{\prime *}\right)=r\left(G^{\prime}\right)=0$, by the induction hypothesis, $o\left(G^{*}\right)=0$. It follows from Lemma 3 that $o(G) \leq o\left(G^{\prime}\right)=o\left(G^{*}\right)=0$. Hence $o(G)=0$.

In the following we assume that for any two edges $e_{1}, e_{2}$ incident to a vertex $v$, at most one of the segments $e_{1} v e_{2}, e_{2} v e_{1}$ is contained in $W$.

We now choose any edge $e=u v$ such that $W=u e v P_{1} v e u P_{2}$ and all the edges of $P_{1}$ are distinct. Note that such an edge exists, we may simply choose $P_{1}$ to be any maximal closed walk which contains each edge at most once. Observe that $P_{1}, P_{2}$ are closed walks which cover all the edges of $G-e$, once in each direction. Moreover $P_{1}, P_{2}$ are retracting free. Indeed, $P_{1}$ is retracting free, as all the edges of $P_{1}$ are distinct. If $P_{2}$ contains a retraction, then the retraction occurs at $u$ and $P_{2}$ is of the form $u e^{\prime} w Z w e^{\prime} u$. This implies that $W$ contains both segments $e u e^{\prime}$ and $e^{\prime} u e$, contrary to our assumption.

First we assume that $d_{G}(v)=3$. Let the other two edges incident to $v$ be $e^{\prime}=v x$ and $e^{\prime \prime}=v y$. Since $P_{1}$ covers each edge of $G$ at most once, it is easy to see that both closed walks $P_{1}, P_{2}$ covers $e^{\prime}, e^{\prime \prime}$. We may assume that $P_{1}=v e^{\prime} x A y e^{\prime \prime} v$ and $P_{2}=v e^{\prime \prime} y B x e^{\prime} v$. Let $W^{\prime}=x A y B x$. Then $W^{\prime}$ is a closed walk which covers all the edges of $G-v$ once in each direction. We shall show that $W^{\prime}$ is retracting free. Indeed, if to the contrary $W^{\prime}$ contains a retraction, then the retraction occurs at $x$ or $y$. Without loss of generality we assume that $W^{\prime}=x e^{*} z C z e^{*} x$ has a retraction 
at $x$. Then $W$ contains both segments $e^{\prime} x e^{*}$ and $e^{*} x e^{\prime}$, contrary to our assumption. Thus $r(G-v)=0$. By the induction hypothesis, we have $o(G-v)=0$. Let $T^{\prime}$ be a spanning tree of $G-v$ with $o\left(T^{\prime}\right)=0$. It is easy to see that $T=T^{\prime}+e$ is a spanning tree of $G$ with $o(T)=0$ in $G$. Therefore $o(G)=0$.

Assume now that $d_{G}(v) \geq 4$. Suppose $P_{1}=v e^{\prime} x A v$ and that $P_{2}=x e^{\prime} v B x$. Let $W^{\prime}=A B$. Then $W^{\prime}$ is a closed walk which covers each of the edges of $G-e-e^{\prime}$ once in each direction. We shall show that $W^{\prime}$ is retracting free. If $W^{\prime}=x e^{\prime \prime} y C y e^{\prime \prime} x$ has a retraction at $x$ then $W$ contains both segments $e^{\prime} x e^{\prime \prime}$ and $e^{\prime \prime} x e^{\prime}$, contrary to our assumption. If $W^{\prime}=v e^{\prime \prime} z C z e^{\prime \prime} v$ contains a retraction at $v$ then we obtain a graph $G^{\prime}$ from $G$ by splitting $v$ into two vertices $v^{\prime}, v^{\prime \prime}$ and by letting $e, e^{\prime}, e^{\prime \prime}$ incident to $v^{\prime}$ and the other edges incident to $v$ be incident to $v^{\prime \prime}$. It is easy to see that $W$ is a retracting free bidirectional double tracing of $G^{\prime}$. By applying the argument of the previous paragraph to $G^{\prime}$ with $v=v^{\prime}$ (observe that $d_{G^{\prime}}\left(v^{\prime}\right)=3$ ), we conclude that $o\left(G^{\prime}\right)=0$, and it follows from Lemma $3(2)$ that $o(G)=0$.

Therefore $W^{\prime}$ is retracting free and hence $r\left(G-e-e^{\prime}\right)=0$. By the induction hypothesis, $o\left(G-e-e^{\prime}\right)=0$. Let $T$ be a spanning tree of $G-e-e^{\prime}$ such that $o(T)=0$. Then since $d_{G}(v) \geq 4$, it follows that $T$ as a spanning tree of $G$ also has $o(T)=0$. Therefore $o(G)=0$.

Next suppose that $o(G)=0$. We shall prove that $G$ has a retracting free bidirectional double tracing by induction on $s(G)=\sum_{d_{G}(v) \geq 3}\left(d_{G}(v)-3\right)$.

Suppose first that $s(G)=0$. Hence $G$ is a cubic graph. We proceed by induction on $|E(G)|$. If $|E(G)|=3$, then the only case that $o(G)=0$ is that $G$ has two vertices and three parallel edges joining them, we denote this graph by $G_{0}$. (As $s(G)=0, G$ contains no vertex of degree greater than 3. The only other connected cubic graph with three edges is $K_{2}$ with a loop on each of the two vertices, and this graph has $o(G)=2$.) It is easy to find a retracting free bidirectional double tracing of $G_{0}$.

Assume now that $G$ is a connected cubic graph, $o(G)=0$ and that for any connected cubic graph $G^{\prime}$ with fewer edges than $G$ and $o\left(G^{\prime}\right)=0$ we have $r\left(G^{\prime}\right)=0$. Let $T$ be a spanning tree of $G$ such that $o(T)=0$, and let $B$ be a non-trivial component of $G-E(T)$, i.e., $B$ contains at least one edge. Then $B$ is either an even cycle or an even path. If $B$ is an even cycle, then let $v$ be any vertex of $B$; 
if $B$ is an even path then let $v$ be the second vertex of $B$ along the path. In any case $d_{B}(v)=2$, hence $d_{T}(v)=1$. Let $T^{\prime}=T-v$. Then $T^{\prime}$ is a spanning tree of $G-v$ and all components of $(G-v)-T^{\prime}$ have even number of edges. Therefore $o\left(T^{\prime}\right)=0$ in $G-v$. If $\delta(G-v) \geq 2$, then $r(G-v)=0$ by the induction hypothesis. (Note that although $G-v$ is not 3-regular, by Lemma 3, we may change $G-v$ into a 3-regular graph $G^{\prime}$ such that $o\left(G^{\prime}\right)=o(G-v)$ and $r\left(G^{\prime}\right)=r(G-v)$ and that $\left|E\left(G^{\prime}\right)\right| \leq|E(G-v)|$.) Applying Lemma 5, we have $r(G)=0$.

If $G-v$ has a vertex, say $u$, of degree 1 , then it must be the case that there are two parallel edges between $u$ and $v$. Denote by $e_{1}, e_{2}$ the two parallel edges between $v$ and $u$, and denote by $e_{3}$ the other edge incident with $u$, and $e_{4}$ the other edge incident with $v$. Let $w$ be the neighbour of $u$ (in $G-v$ ), and let $z$ be the other neighbour of $v$. If $w \neq z$, then it is straightforward to verify that $T-u-v$ is a spanning tree of $G-u-v$ with $o(T-u-v)=0$. Hence $o(G-u-v)=0$. By the induction hypothesis, $r(G-u-v)=0$. Let $W$ be a retracting free bidirectional double tracing of $G-u-v$. Assume that $W=A w B z$. Then

$$
W^{\prime}=A w e_{3} u e_{1} v e_{2} u e_{3} w B z e_{4} v e_{1} u e_{2} v e_{4} z
$$

is a retracting free bidirectional double tracing of $G$. If $w=z$, then let $y$ be the other neighbour of $w$ (i.e., the one different from $u$ and $v$ ), and let $e_{5}=w y$. It is easy to verify that $T-u-v-w$ is a spanning tree of $G-u-v-w$ with $o(T)=0$. Hence by the induction hypothesis, $G-u-v-w$ has a retracting free bidirectional double tracing $W$. Assume $W=A y$. Then

$$
W^{\prime}=A_{y e} e_{3} u e_{1} v e_{2} u e_{3} w e_{4} v e_{1} u e_{2} v e_{4} w e_{5} y
$$

is a retracting free bidirectional double tracing of $G$.

Now suppose $s(G)=k>0$ and that $r\left(G^{\prime}\right)=0$ for all graphs $G^{\prime}$ for which $o\left(G^{\prime}\right)=0$ and $s\left(G^{\prime}\right)<k$. Similarly let $T$ be a spanning tree of $G$ such that $o(T)=0$. Let $v$ be a vertex of $G$ with $d_{G}(v) \geq 4$, and let $B$ be the component of $G-E(T)$ which contains $v$. If $B$ is a singleton, then let $G^{\prime}$ be obtained from $G$ by splitting $v$ into two vertices $v^{\prime}, v^{\prime \prime}$ and connecting $v^{\prime}, v^{\prime \prime}$ by an edge. The edges incident to $v$ are arbitrarily distributed among $v^{\prime}$ and $v^{\prime \prime}$ so that $d_{G^{\prime}}\left(v^{\prime}\right), d_{G^{\prime}}\left(v^{\prime \prime}\right) \geq 3$. Let $T^{\prime}=T+v^{\prime} v^{\prime \prime}$. Then $T^{\prime}$ is a spanning tree of $G^{\prime}$ with $o\left(T^{\prime}\right)=0$ in $G^{\prime}$. Since 
$s\left(G^{\prime}\right)<s(G)$, by the induction hypothesis, $r\left(G^{\prime}\right)=0$. It follows from Lemma 3 that $r(G)=0$.

Secondly assume that $d_{B}(v)=1$. Let $e=v u \in B$ and let $e^{\prime}=v w \in E(T)$ be the edge contained in the (unique) cycle of $T+e$. Let $G^{\prime}$ be obtained from $G$ by splitting $v$ into two vertices $v^{\prime}, v^{\prime \prime}$ and connecting $v^{\prime}, v^{\prime \prime}$ by an edge. The edges incident to $v$ are arbitrarily distributed among $v^{\prime}$ and $v^{\prime \prime}$, so that $d_{G^{\prime}}\left(v^{\prime}\right), d_{G^{\prime}}\left(v^{\prime \prime}\right) \geq 3$ and that $e, e^{\prime}$ are incident to $v^{\prime}, v^{\prime \prime}$ respectively. If $|E(B)|$ is even, then let $T^{\prime}=T+v^{\prime} v^{\prime \prime}$. It is easy to see that $T^{\prime}$ is a spanning tree of $G^{\prime}$ with $o\left(T^{\prime}\right)=0$ in $G^{\prime}$. Since $s\left(G^{\prime}\right)<s(G)$, by the induction hypothesis, $r\left(G^{\prime}\right)=0$. It then follows from Lemma 3 that $r(G)=0$. If $|E(B)|$ is odd, then let $G^{\prime \prime}$ be obtained from $G^{\prime}$ by deleting the edge $v^{\prime} v^{\prime \prime}$, and let $T^{\prime}=T+e$. Again $T^{\prime}$ is a spanning tree of $G^{\prime \prime}$ with $o\left(T^{\prime}\right)=0$ in $G^{\prime \prime}$. By induction hypothesis, $r\left(G^{\prime \prime}\right)=0$. It follows from Lemma 3 that $r(G)=0$.

Thirdly assume that $d_{B}(v) \geq 2, d_{T}(v) \geq 2$. If $B$ is a single loop edge $e$, then let $T^{\prime}=T, G^{\prime}=G-e$. Then $o\left(T^{\prime}\right)=0$ in $G^{\prime}$ and $r\left(G^{\prime}\right)=0$ by the induction hypothesis. Let $W$ be a retracting free bidirectional double tracing of $G^{\prime}$, and let $W_{1}, W_{2}$ be the loop on $v$ traversed in opposite directions. Then $\left\{W, W_{1}, W_{2}\right\}$ is a retracting free oriented walk double covering of $G$. By Lemma 2, these three walks can be combined into a single retracting free closed walk, which is a retracting free bidirectional double tracing of $G$. Hence $r(G)=0$. If $B$ is not a single loop edge, then let $G^{\prime}$ be the graph obtained from $G$ by splitting $v$ into two vertices $v^{\prime}, v^{\prime \prime}$, and let all the edges of $B$ incident to $v$ be incident to $v^{\prime}$ and all edges of $T$ incident to $v$ be incident to $v^{\prime \prime}$, and connect $v^{\prime}, v^{\prime \prime}$ by an edge. If $|E(B)|$ is even, then let $T^{\prime}=T+v^{\prime} v^{\prime \prime}$. It is easy to see that $T^{\prime}$ is a spanning tree of $G^{\prime}$ for which $o\left(T^{\prime}\right)=0$ in $G^{\prime}$. Therefore $r\left(G^{\prime}\right)=0$ by induction hypothesis (as $s\left(G^{\prime}\right)<s(G)$ ) and it follows from Lemma 3 that $r(G)=0$. If $|E(B)|$ is odd, then let $G^{\prime \prime}$ be obtained from $G^{\prime}$ by deleting the edge $v^{\prime} v^{\prime \prime}$, and let $T^{\prime}=T+e^{\prime}$ where $e^{\prime} \in B$ is an edge incident to $v$ (thus incident to $v^{\prime}$ in $G^{\prime \prime}$ ) for which $B-e^{\prime}$ is either connected or consists of two parts, each has an even number of edges. (It is easy to see that at least one of the edges of $B$ incident to $v$ can be chosen as $e^{\prime}$.) Now $T^{\prime}$ is a spanning tree of $G^{\prime \prime}$ with $o\left(T^{\prime}\right)=0$ in $G^{\prime \prime}$. Again by induction $r\left(G^{\prime \prime}\right)=0$, and it follows from Lemma 3 that $r(G)=0$. 
Finally assume that $d_{T}(v)=1$. Then $d_{B}(v) \geq 3$, let $G^{\prime}$ be the graph obtained from $G$ by splitting $v$ into two adjacent vertices $v^{\prime}, v^{\prime \prime}$ such that two edges of $B$ incident to $v^{\prime}$ and all other edges incident to $v^{\prime \prime}$. The two edges of $B$ incident to $v^{\prime}$ are chosen in such a way that $\left|E\left(B^{\prime}\right)\right|$ has the same parity as $|E(B)|$, where $B^{\prime}$ is the component of $B$ formed by the splitting and containing $v^{\prime}$. It is easy to see that such a splitting exists. Indeed, if there exists a splitting such that $B$ becomes $B^{\prime}$, i.e., all the edges of $B$ are still in the same connected component, then this is true, as $B^{\prime}$ and $B$ consist of the same set of edges. Otherwise each edge of $B$ incident with $v$ is a cut edge of $B$. Then it is easy to choose a splitting satisfying the conditions.

If $|E(B)|$ is even, then let $G^{\prime \prime}=G^{\prime}, T^{\prime}=T+v^{\prime} v^{\prime \prime}$. It is easy to see that $o\left(T^{\prime}\right)=0$ and hence $o\left(G^{\prime}\right)=0$. By the induction hypothesis $r\left(G^{\prime}\right)=0$, and it follows from Lemma 3 that $r(G)=0$.

If $|E(B)|$ is odd, then let $e \in E\left(B^{\prime}\right)$ be an edge such that $B^{\prime}-e$ is either connected or contains two components of even number edges. Let $G^{\prime \prime}=G^{\prime}-v^{\prime} v^{\prime \prime}$ and $T^{\prime}=T+e$. Then $T^{\prime}$ is a spanning tree of $G^{\prime \prime}$ with $o\left(T^{\prime}\right)=0$ in $G^{\prime \prime}$. Again by the induction hypothesis, $r\left(G^{\prime}\right)=0$, and it follows from Lemma 3 that $r(G)=0$. This completes the proof of Theorem 1 .

By Theorem 1 and the proof above, we obtain a structure theorem concerning graphs $G$ with $r(G)=0$.

Definition 1 Suppose $G$ is a graph and $u, v$ are vertices of $G$. When we say add $a$ bond bridge between $u$ and $v$ we mean the addition of two new vertices $x$ and $y$ and the following four edges: two parallel edges between $x$ and $y$, one edge between $x$ and $u$, and one edge between $v$ and $y$.

When we say add a bud at vertex $u$ we mean the addition of three new vertices $x, y$ and $z$, and the following five edges: two parallel edges between $x$ and $y$, one edge between $x$ and $z$, one edge between $y$ and $z$, and one edge between $z$ and $u$.

In the alternative proof of Theorem 1 (the part for the case that $s(G)=0$ ), we actually proved that if $r(G)=0$, then the addition of a bond bridge or the addition of a bud, result in a graph $G^{\prime}$ with $r\left(G^{\prime}\right)=0$. Lemma 5 shows that the addition 
of a vertex of degree 3 result in a graph $G^{\prime}$ with $r\left(G^{\prime}\right)=0$. Lemma 3 shows that subdivision of an edge, contracting an edge, identifying two nonadjacent vertices of $G$ will also result in a graph $G^{\prime}$ with $r\left(G^{\prime}\right)=0$. Our next result asserts that all graph $G^{\prime}$ with $r\left(G^{\prime}\right)=0$ can be constructed from $G_{0}$ by a series of these operations.

Theorem 4 Suppose $G$ is a connected graph. Then $r(G)=0$ if and only if $G$ can be constructed from $G_{0}$ through a series of subdivision and adding 3-vertex, bond bridge or bud, and finally contracting edges and identifying vertices.

Proof. We have already shown that all such constructed graphs $G$ have $r(G)=0$.

Conversely, suppose $r(G)=0$. Then $\delta(G) \geq 2, o(G)=0$ by Theorem 1 . If there is a vertex $v \in V(G)$ such that $d_{G}(v)>3$, by the second part of the proof of Theorem 1 given above, we can split $v$ into two adjacent or nonadjacent vertices $v^{\prime}, v^{\prime \prime}$ such that the obtained graph $G^{\prime}$ satisfies $o\left(G^{\prime}\right)=0,2 \leq d_{G^{\prime}}\left(v^{\prime}\right)<d_{G}(v), 2 \leq$ $d_{G^{\prime}}\left(v^{\prime \prime}\right)<d_{G}(v)$. Note that the inverse operation of splitting is contracting an edge or identifying two vertices according to $v^{\prime}$ and $v^{\prime \prime}$ are adjacent or not. Continue the splitting, we finally obtained a graph $G_{1}$ with $2 \leq \delta\left(G_{1}\right) \leq \Delta\left(G_{1}\right) \leq 3, o\left(G_{1}\right)=0$. Then Contract the edges incident with a degree 2 -vertex one by one, we obtain a cubic graph $G_{2}$ with $o\left(G_{2}\right)=0$. Again by the the proof of Theorem 1, we can delete a degree 3 -vertex, a bond bridge or a bud to obtain a graph $G_{3}$ with $o\left(G_{3}\right)=0,2 \leq \delta\left(G_{3}\right) \leq \Delta\left(G_{3}\right) \leq 3, n\left(G_{3}\right)<n\left(G_{2}\right)$. Continue this process, we will finally obtain $G_{0}$. Inverse the process, we conclude that $G$ can be obtained from $G_{0}$ through a series of subdivisions, adding degree 3 -vertices, adding bond bridges or buds, and finally contracting edges and identifying vertices.

Theorem 5 Let $G$ be a connected non-cycle graph with $\delta(G) \geq 2$, then $r(G)=$ $o(G)$.

Proof. By Theorem 1, we may assume that $r(G)>0, o(G)>0$. By Lemma 3 , we may assume that $\delta(G) \geq 3$. We shall prove this theorem by induction on $|E(G)|$. If $|E(G)| \leq 3$, then it can be easily verified that the theorem is true. Suppose the theorem is true for all graphs $G^{\prime}$ with $\left|E\left(G^{\prime}\right)\right| \leq k$ and that $G$ is a 
graph with $|E(G)|=k \geq 4$. Let $W$ be a bidirectional double tracing of $G$ with minimum number of retracings. Similar to the alternative proof of Theorem 1 , we may assume that there are no edges $e_{1}, e_{2}$ of $G$ such that $W$ is of the form $e_{1} v e_{2} P_{1} e_{2} v e_{1} P_{2}$. Let $e=u v$ be a retracting edge of $W$ retracting at $v$. Now delete $e$ in $W$, we obtain a bidirectional double tracing $W^{\prime}$ of $G-v$ with $r\left(W^{\prime}\right)=r(W)-1$ so that $r(G-e) \leq r(G)-1$. Then by the induction hypothesis and Lemma 3, $o(G)-1 \leq o(G-e)=r(G-e) \leq r(G)-1$, hence $o(G) \leq r(G)$. Conversely, let $e$ be an edge in an odd component $B$ of $G-E(T)$, for which $o(T)=o(G)$ and $B-e$ is connected. Then delete $e$ we have $o(G-e) \leq o\left(T^{\prime}\right)=o(T)-1 \leq o(G)-1$, and then $r(G)-1 \leq r(G-e)=o(G-e) \leq o(G)-1$. Therefore $o(G)=r(G)$.

Theorem 6 There is a polynomial time algorithm to determine $r(G)$.

Proof. By Lemma 3, we may assume that $\delta(G) \geq 3$.

Let $H$ be the subgraph of $G$ induced by the vertices of degree 3. For each edge $e=u v$ of $G$, we associate a subset $E(e)$ of $E(G)$ as follows: if $\{u, v\} \cap V(H)=\varnothing$, then $E(e)=\left\{e^{\prime} \in E(G): e^{\prime} \neq e, e^{\prime} \notin E(H)\right\}$; if $\{u, v\} \subset V(H)$, then $E(e)=$ $\left\{e^{\prime} \in E(G): e^{\prime}\right.$ is incident with $\left.e\right\}$; if $\{u, v\} \cap V(H) \neq \varnothing$ and $\{u, v\} \not \subset V(H)$, then $E(e)=\left\{e^{\prime} \in E(G): e^{\prime} \notin E(H)\right.$ or $e^{\prime}$ is incident with $\left.e\right\}$;

Let $G^{\prime}$ be the graph obtained from $G$ by subdividing each edge $e=u v$ such that it becomes a path, say $P_{e}$, of length $|E(e)|$. We label each of the $|E(e)|$ edges in the path $P_{e}$ by one of the labels $\left\{e, e^{\prime}\right\}, e^{\prime} \in E(e)$. Thus each edge of $G^{\prime}$ is labeled with an unordered pair of edges of $G$, and each label appears twice among the edges of $G^{\prime}$. We say that the two edges with the same label are partners. For a spanning tree $T^{\prime}$ of $G^{\prime}$, we denote by $t\left(T^{\prime}\right)$ the number of edges $e$ in $T^{\prime}$ whose partners are not edges of $T^{\prime}$, and let $t\left(G^{\prime}\right)=\min \left\{t\left(T^{\prime}\right): T^{\prime}\right.$ is a spanning tree of $\left.G^{\prime}\right\}$. We show that $t\left(G^{\prime}\right)=o(G)$ when $|E(G)|-|V(G)|-o(T)+1$ is even, and otherwise $t\left(G^{\prime}\right)=o(G)+1$.

Let $T^{\prime}$ be a spanning tree of $G^{\prime}$ with $t\left(T^{\prime}\right)=t\left(G^{\prime}\right)$. Let $T$ be the subgraph of $G$ induced by the edge set $E(T)=\left\{e: P_{e} \subset E\left(T^{\prime}\right)\right\}$. It is easy to verify that there is at least one edge $e$ in each odd component of $G-E(T)$ and another edge $e^{\prime}$ such that only one of the edges with label $\left\{e, e^{\prime}\right\}$ is in $T^{\prime}$, and when $|E(G)|-|V(G)|-o(T)+1$ 
is odd, there is at lesat one edge in the non-odd component of $G-E(T)$ with above property. Therefore $o(T) \leq t\left(T^{\prime}\right), o(G) \leq t\left(T^{\prime}\right)=t\left(G^{\prime}\right)$, and when $|E(G)|-|V(G)|-$ $o(T)+1$ is odd, $o(T) \leq t\left(T^{\prime}\right)-1$, thus $o(G) \leq t\left(T^{\prime}\right)-1=t\left(G^{\prime}\right)-1$.

Conversely, for a spanning tree $T$ of $G$ with $o(T)=o(G)$. It is not difficult to see that we can pair off a subset of the edges of $E(G)-E(T)$ in such a way that, when $|E(G)|-|V(G)|-o(T)+1$ is even, exactly $o(T)$ edges are not paired with other edges; otherwise exactly $o(T)+1$ edges are not paired with other edges, and each pair of edges $\left\{e, e^{\prime}\right\}$ satisfies $e \in E\left(e^{\prime}\right)$, and hence $e^{\prime} \in E(e)$. We delete all the edges of $G^{\prime}$ whose labels are those paired edges $\left\{e, e^{\prime}\right\}$, and for those $e \in E(G)-E(T)$ which is not paired with other edges, we arbitrarily delete an edge from the path $P_{e}$. Then we obtain a spanning tree $T^{\prime}$ of $G^{\prime}$ with $t\left(T^{\prime}\right)=o(T)=o(G)$ or $t\left(T^{\prime}\right)=$ $o(T)+1=o(G)+1$. Therefore when $|E(G)|-|V(G)|-o(T)+1$ is even, $t\left(G^{\prime}\right) \leq o(G)$, hence $t\left(G^{\prime}\right)=o(G)$. When $|E(G)|-|V(G)|-o(T)+1$ is odd, $t\left(G^{\prime}\right) \leq o(G)+1$, then $t\left(G^{\prime}\right)=o(G)+1$.

Now we know that $o(G)$ can be determined by $t\left(G^{\prime}\right)$. Since $t\left(G^{\prime}\right)=n\left(G^{\prime}\right)-2 \nu$, where $\nu$ is the size of the maximum matching of the linear 2-polymatroid formed by the pairs of $G^{\prime}$ and the rank function of graph, and it is shown in [4], [5] that the $\nu$ can be determined in polynomial time of the size of $G^{\prime}$. Therefore we can determine $o(G)$ in time polynomial of the size of $G$.

The proof of Theorem 6 is essentially the one presented in [8]. However there was a minor error in the algorithm presented in [8]. In that algorithm, the set $E(e)$ associated to edges $e=u v$ for which $\{u, v\} \not \subset V(H)$ are equal to $E(G)-\{e\}$. (The rest is the same as the algorithm presented here, except that the algorithm in [8] only tells whether $o(G)=0$.) It is easy to see that thus constructed graphs $G^{\prime}$ always have $t\left(G^{\prime}\right)=0$, provided that $|E(G)|-|V(G)|+1$ is even (which is assumed).

We close this paper with two open questions:

(1): Even though there is a polynomial time algorithm for determining $r(G)$, as shown by Theorem 6 , the running time is about $O\left(n^{40}\right)$, which is certainly not efficient. This is due to fact that the best known algorithm for finding the maximum matching in a linear 2-polymatroid is $O\left(n^{\prime 10}\right)$ [5]. It is probably worthy to find a direct algorithm for determining $r(G)$ and for finding a bidirectional double tracing 
with nimimum number of retractings.

(2): The authors of this paper came across the problem of bidirectional double tracing by considering the so called "garbage collecting" problem, where a garbage collecting truck needs to traverse each side of every street exactly once, making as few U-turns (retractions) as possible. In practical situation, for narrow streets (or backyard streets), the truck may just traverse the street once in either direction. Thus we have a graph $G$ and a subset $E^{\prime} \subset E(G)$. We need to find a closed walk of $G$ that traverses each of the edges in $E^{\prime}$ once in either direction, and traverses all the other edges once in each direction. Such a closed walk exists if and only if all degrees of the graph induced by $E^{\prime}$ are even [3]. In case such a walk exists, our goal is also to minimize the number of retractions of the closed walk. Denote by $r\left(G ; E^{\prime}\right)$ the minimum number of retractions in such a closed walk. How to determine the parameter $r\left(G ; E^{\prime}\right)$ remains an open problem. In case such a closed walk does not exist, one problem is to find a closed walk that traverses each edge in the right direction at least as many times as required, and which has the shortest total length. The case $E^{\prime}=E(G)$ of this problem is exactly the Chinese postman problem. When $E^{\prime} \neq E(G)$, then because of the directions of the edges involved, it is different from the Chinese postman problem. In the objective function, we may also combine the total length of the closed walk and the number of retractings together, which might result in some interesting problems.

Acknowledgment. This work was done when the authors were visiting Simon Fraser University. The authors would like to express their sincere thanks to Professors B. Alspach, P. Hell and L. Goddyn for their help and for bringing the references to their attention.

\section{References}

[1] J.A. Bondy and U.S. Murty, Graph Theory with Applications, Macmillan, London(1976).

[2] R.B. Eggleton and D. K. Skilton, Double tracings of graphs, Ars Combin. 
A(1984), 307-323.

[3] H.Fleischner, Eulerian Graphs and Related Topics Vol.2, Annals of Discrete Mathematics 50, North-Holland 1991.

[4] L. Lovasz, The matroid matching problem, Algebraic Methods in Graph Theory, Colloquia Mathematica Societatis Janos Bolai, Szegd, Hungary, 1978.

[5] L. Lovasz and M.D. Plummer, Matching Theory, North-Holland, 1986.

[6] O. Ore, A problem regarding the tracing of graphs, Elem. Meth. 6 (1951), 49-53.

[7] D.K.Skilton, On the retracting number of graph, Research Report of SFU (1985)9.

[8] C.Thomassen, Bidirectional Retracting-Free Double Tracings and Upper Embeddability of Graphs, J. Combinatorial Theory, Ser. (B) 50(1990) 198-207. 